\title{
Competence networks in the era of CPS - lessons learnt in the ICES cross-disciplinary and multi-domain center
}

\author{
Martin Törngren ${ }^{1}$, Fredrik Asplund ${ }^{1}$, Tor Ericson², Catrin Granbom³ ${ }^{3}$ Erik Herzog ${ }^{4}$, Zhonghai Lu1 ${ }^{1}$, Mats Magnusson ${ }^{1}$, Maria Månsson ${ }^{5}$, \\ Stefan Norrwing ${ }^{1,5}$, Johanna Olsson ${ }^{1}$, and Johnny Öberg ${ }^{1}$. \\ ${ }^{1}$ KTH Royal Institute of Technology, 10044 Stockholm, Sweden \\ 2 ÅF Digital Solutions AB, 16999 Stockholm, Sweden \\ ${ }^{3}$ ERICSSON AB, Torshamnsg. 21-23, 16480 Stockholm, Sweden \\ ${ }^{4}$ Saab Aeronautics, 58188 Linköping, Sweden \\ ${ }^{5}$ Prevas AB, 12030 Stockholm, Sweden
}

\begin{abstract}
Cyber-Physical Systems (CPS) are evolving to become more intelligent, autonomous and collaborating, playing an important role in societal infrastructure. The amount of knowledge required in developing and managing future CPS will be unprecedented, leading to stronger needs for collaboration, competence provisioning, continuous learning and renewal of education. This is where "competence" (or learning) "networks" involving academia and industry play an important role. We elaborate and discuss needs, lessons learnt and challenges for such competence networks in the context of CPS. We draw upon our experiences gained from ICES - the KTH-industry crossdisciplinary and multi-domain competence network which in 2019 has been operational for 11 years, growing from 6 to more than 30 participating organizations. The ICES network focuses on activities to support students, industrial engineers and managers, and academic faculty, acting as a network, catalyst and competence provider directed towards these stakeholders. We elaborate challenges faced during the operation of ICES including the lack of prioritization of competence networks and education, the paradox with strong needs for competence networks but perceived lack of time, the challenges of reaching out to stakeholders, and fragmented efforts addressing competence provisioning. We finally discuss ways forward. In conclusion, we believe that the ICES type of network could be relevant in many other areas characterized by complex systems.
\end{abstract}

Keywords. Cyber-physical systems, Embedded systems, Competence networks, Learning networks, Industry-academia collaboration, Engineering education, Technological paradigm shifts, Life-long learning, science outreach

\section{Introduction and motivation}

Enabled by continued technological advances, Cyber-Physical Systems (CPS) are evolving to become more intelligent, autonomous and interconnected, and with an increasing collaboration with other systems. As a result, CPS are being (and will be) deployed in all kinds of applications in society, playing an important role in societal infrastructure. These trends represent what many see as a socio-technical paradigm shift in which present engineering methodologies and legal frameworks will have to change to accommodate for the new systems built. And paradigm shifts do take time, [30]! A typical example is that of intelligent transportation systems involving highly automated vehicles. For such systems, neither engineering methodologies to ensure safety and availability, nor societal frameworks (e.g. for liability, homologation and insurances) are in place. New ground has to be broken $[1,2]$.

The corresponding capabilities and complexity of future CPS will generally demand much more from various stakeholders, in particular concerning CPS development, operational management and maintenance. This will involve more and more experts and knowledge. Even large organizations will face challenges in incorporating all expertise needed, implying that the CPS supply chain and usage will evolve further towards sophisticated networks of collaborating organizations, ranging from customers to CPS component manufacturers.

The engineering of CPS has always been a multidisciplinary endeavor. However, there is a growing scope of concerns that needs to be addressed, such as sustainability, complex unstructured environments, technological content (e.g. AI and cloud connectivity), systems of systems collaboration, system change (due to updates and learning), and new and changing risks (e.g. cyber-security, safety and privacy concerns).

These new trends, supply chains and concerns, paired with a faster development speed, result in a situation in which competence provisioning and life-long learning for organizations working with and using CPS becomes crucial. The need for what we refer to as competence or learning networks is therefore growing. With a competence network, we refer primarily to nonprofit collaborations to promote learning and knowledge creation. While the concept of a competence network is general, we use it here in the specific context of the engineering of CPS and related education and research.

We elaborate and discuss needs, lessons learnt and challenges for such competence networks in the context of embedded and cyber-physical systems. We draw upon our experiences gained from ICES (Innovative Center for Embedded Systems) - the 
KTH-industry cross-disciplinary and multi-domain competence network. In 2019 ICES has been operational for 11 years, and has during this period grown from 6 to more than 30 participating organizations, [3, 4]. The ICES network focuses on activities to support students, industrial engineers, managers, and academic faculty as key stakeholders. The network further has a focus on shared concerns among members across industrial domains - it acts as a network, catalyst and competence provider directed towards the key stakeholders, while collaborating with other networks.

The paper is organized as follows. In Section 2 we briefly review the state of the on competence networks and other initiatives with a flavor of "competence network". In Section 3 we describe the ICES competence network and its developed practices. In Section 4 we highlight lessons learnt, aspects that we believe are important in organizing and operating competence networks. We correspondingly then in Section 5 turn to challenges that we have identified over the years - issues that need to be handled and overcome for a competence network to function. In Section 6 we discuss the findings and their potential generalization - the relevance of the identified lessons learnt and challenges in other settings. Finally, in Section 7, we present conclusions and ways forward.

\section{State of the art and other networks}

In this section we first describe research that relates to competence and learning networks. We then briefly outline a number of existing initiatives with some ingredients of competence provisioning.

\subsection{Competence networks: State of the Art}

There is no extensive discourse on competence networks, or non-profit collaborations to promote learning, per se. However, there exists a wider discussion on (public-private) networks and the future of engineering education.

As knowledge can be seen as a resource that enables firms to stay competitive, [15], network cooperation can be seen as beneficial by generating complementarities and inter-firm learning, [16, 17]. It is typically also an efficient way to share knowledge as the associated breadth of competencies can be exploited over a long time period. In regard to different types of networks those that contain both industry and academia are often of special interest, as firms can use them to access new technology and specialized consultancy, [18]. However, academic institutions can also benefit from such networks when covering the whole state of the art of a topic area is beyond any single institute - they allow academics to identify important topics, [19], and to understand exploitation better, [20].

As far as the state of the art is concerned there is thus a heavy emphasis on studies concerning knowledge transfer from, or services provided by, academia to industry. The implications of this relationship on academia as a whole has not received as much attention.

However, these interactions are not effortless. Organizations do not have an easy time engaging with organizations that are not similar to them due to difficulties to identify and absorb knowledge that is different from their own, [21]. This is by necessity the case for academic institutions and firms. At the same time, firms typically do not get as much value capture (in terms of innovations, as represented by e.g. patents) from cooperation with academia when they also invest heavily in internal scientific research, [22, 23]. Academic institutions are similarly negatively affected - the broader a university's collaboration breadth, the more negative increasing cooperation with industry reflects on academic innovation, [20].

The cost of engaging in a competence network might thus be considerable, while potentially neither resulting in a direct monetary benefit to industry nor academia. However, if a firm and academic institute are indeed closely aligned, the former's recruitment of employees should regardless benefit from a close cooperation.

On that note engineering programs are currently tasked with fostering new skills, such as cultural awareness, sustainability, innovativeness, entrepreneurship, etc., [24]. This is driven by demand from firms and the opening up of new career paths, [25]. However, with engineering curricula already stretched to their limits, the introduction of new content is difficult. Both new teaching practices and learning environments have been suggested as solutions to this dilemma, [24]. However, while teachers in higher education might not be outright dismissive of these suggestions, they often have a blind spot in regard to them. This is unfortunate, as new learning environments can lend themselves naturally to new pedagogies. A solution to this problem might be an increased societal support for lifelong learning, where the professional environment of learners can be matched to new learning approaches.

The challenges of creating viable lifelong learning opportunities will still be significant, [25]. However, even if firms do not see direct payback in the form of innovative products from their cooperation with academia, they could thus benefit from forming alliances to coordinate lifelong learning. If they can match their specific internal context to the right pedagogies, then they could ensure that new employees had a natural way of progressing from novice to the type of expert they need. 
Furthermore, curricula are affected by occurrences at the societal level, [26]. Downey and Lucena for instance mention the active push by engineering reformers in the US to increase the importance of scientific skills in engineering education following the Sputnik incident, [27]. This revamping of US engineering education was at least partly to maintain the status of engineering. The emergence of the European Higher Education Area (EHEA) also strongly emphasized supporting peaceful interaction between societies, democracy building and academic freedom, [28]. It was stressed that education should not be considered merely a servant to the purposes of political or economic power. This perspective was challenged during the end of the 1980 's when the cooperation between European universities was being framed as vocational training. This prompted the signing of the Magna Charta Universitatum by heads of universities from all over Europe and beyond, [29].

Clearly the business needs of engineering firms can be both synergetic and in opposition to the wider role of academia. Competence networks could be a more neutral type of cooperation than those focused strongly on value capture, functioning as so called communities of practice, see e.g. $[33,34]$. This could allow industry and academia to achieve a mutual understanding of each other's motivations more easily.

Research centers can also act as competence networks as described in the Berkeley research center experience, highlighting success stories from several research centers with success factors including multidisciplinary (and regionally local) collaboration involving demonstrators, [32].

\subsection{Other related initiatives/networks}

The need for sharing and improving competence is acknowledged and supported by many organizations. In the scope of CPS, we have identified several types of initiatives. The list is not intended to be exhaustive, but to provide representative examples in an attempt to relate ICES to other efforts.

A distinguishing characteristic of ICES in relation to the listed initiatives is that both engineering and $\mathrm{PhD}$ students are found among ICES' stakeholders. This enriches the interactions with the other groups of stakeholders. Most of the other efforts focus on engineers and/or faculty. ICES is also characterized by its regional scope and by being based at a University.

We identified the following types of initiatives, here characterized in terms of primary stakeholders, activities and scope:

- Academic disciplinary communities, for example represented by ESWeek and CPSWeek:

- Primary stakeholders: Academic faculty

- Activities: Academic conferences

- Scope: Worldwide

- Communities for open source software and software forums:

○ Primary stakeholders: Practicing engineers

- Activities: Shared software and discussions hosted by IT platforms.

- Scope: Worldwide

- $\quad$ Social and networks, e.g. Linkedin and Meetup:

- Primary stakeholders: Anyone

- Activities: Dialogues and information sharing hosted by IT-platforms.

- Scope: Worldwide

- Professional associations, e.g. INCOSE and IEEE:

- Primary stakeholders: Association members, typically including industry and academia.

- Activities: Workshops, meetings, roadmapping and project funding (e.g. Artemis-IA/ECSEL).

- Scope: Depends on the scope of the association, e.g. worldwide or Europe.

- Company internal competence networks:

- Primary stakeholders: Company employees

- Activities: Courses, workshops and demonstrator projects.

- Scope: Organization (e.g. company)

- Innovation activities such as EC funded projects, training networks and strategic innovation programs:

- Primary stakeholders: SMEs, innovators, innovation hubs, academia and industry.

- Activities: Innovation projects (acting as "program offices" for setting up projects) and "Innovative Training Networks" (a type of EU funded project that funds $\mathrm{PhD}$ students in multiple countries, involving academia and industry collaboration as well as mobility).

- Scope: According to program/project scope. 
- $\quad$ Networks of excellence (e.g. those previously funded by the EU):

- Primary stakeholders: Academic faculty

- Activities: Workshops, conferences, roadmaps and information provisioning.

- Scope: The network and affiliated partners.

- Traditional research centers hosted by universities:

- Primary stakeholders: Academic faculty and industrial partners (if any).

- Activities: Research projects and workshops.

- Scope: According to center scope.

- Educational initiatives such as Udacity, and EdX:

○ Primary stakeholders: Anyone (e.g. students and practicing engineers)

- Activities: Online courses

- Scope: Global

\section{The ICES competence network}

In 2006 discussions to improve collaboration in the area of embedded systems started within the KTH faculty. Fragmentation was identified during these discussions, in particular in terms of non-collaborating research groups (see Fig. 1) with piecewise contacts to industry in different industrial domains. There was no focused embedded systems program and industry seeking contact with experts had problems finding their way into KTH.

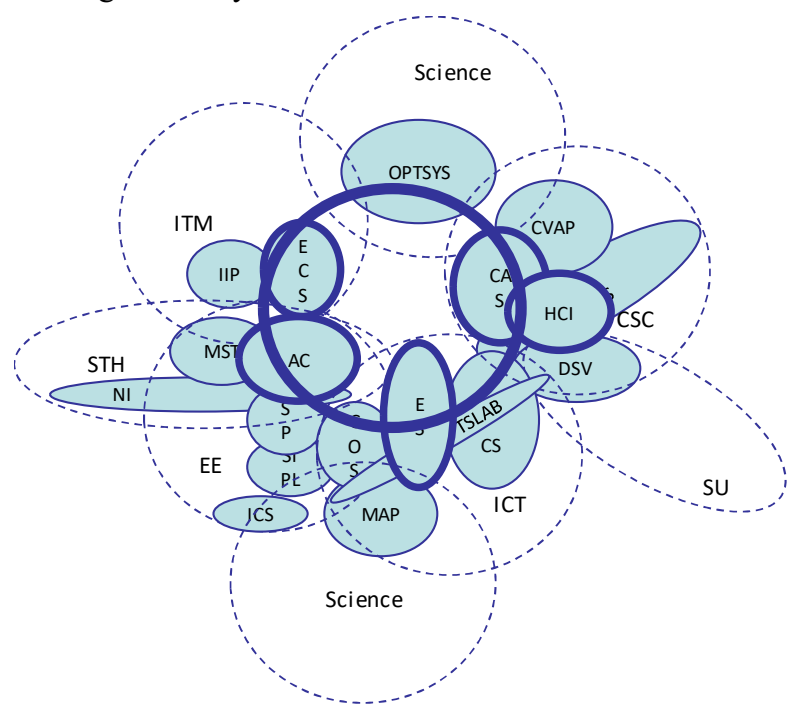

Fig. 1: Initial inventory of KTH groups (and their organizational belonging - the acronyms refer to schools/ departments/ divisions at KTH in 2008), working with some aspects of embedded systems - illustrating the fragmented situation before ICES.

Key stakeholders and role of the network: In discussions involving industry, the suggested solution was to gather resources from all relevant areas in KTH and companies with an interest in research and education in the area of embedded systems as a KTH center. At KTH, a "center" is an entity typically used to cater for cross-school collaboration that also involves external organizations. When ICES was created, the needs and concerns of the various partners and research groups were elicited. This resulted in the realization that while faculty wanted to do research, the industrial partners stated clearly that their main problem was in competence provisioning in a broad sense. They wanted to recruit students, and get access to KTH faculty. As a result, the center was formed as a "competence network", with the purpose to create a platform for contacts among faculty, students and industry. This would allow for exchanging experiences, agreeing on challenges and developing cooperation projects to influence research and education. The key stakeholders of ICES and their identified concerns are shown in Fig. 2.

Thematic focus and goals: The need to understand what the various organizations and researchers considered as relevant topics in the scope of embedded systems surfaced early. After multiple workshops, a view of shared concerns for embedded systems was created. This view was updated during 2017 (see Fig. 3). Only minor changes had to be made during the update. The changes included introducing more industrial domains (as represented by the larger set of member companies) and a larger thematic scope (from embedded systems to encompassing software-intensive CPS). Within this scope, the guiding vision of ICES is to achieve a prospering eco-system for industry and academia, catalyzing world-class education, research and innovation. 


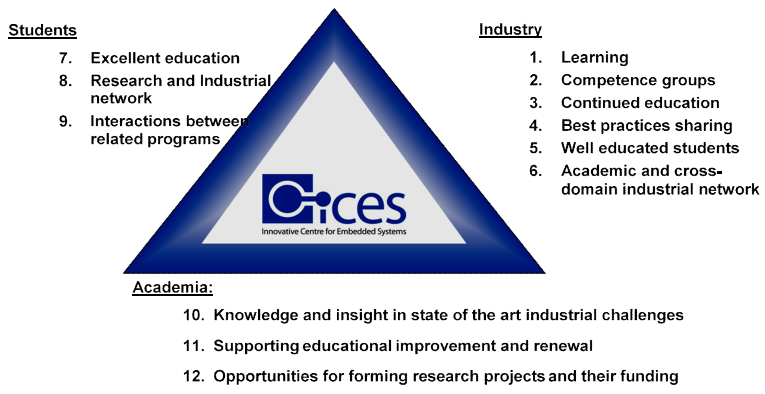

Fig. 2: ICES key stakeholders and their needs

The Vision and Goals document has been revised several times over the years, [4]. It forms an important element of the network. The document describes the long- and short-term goals of ICES, including vision, strategic objectives and operational goals. The strategic objectives include (i) a focus on key concerns that are shared among members across industrial domains (recall Fig. 3), (ii) acting as a network, catalyst and competence provider directed towards the key stakeholders of the center (recall Fig. 2), and (iii) creating synergies and leveraging existing efforts, including with related KTH research centers and other organizations.

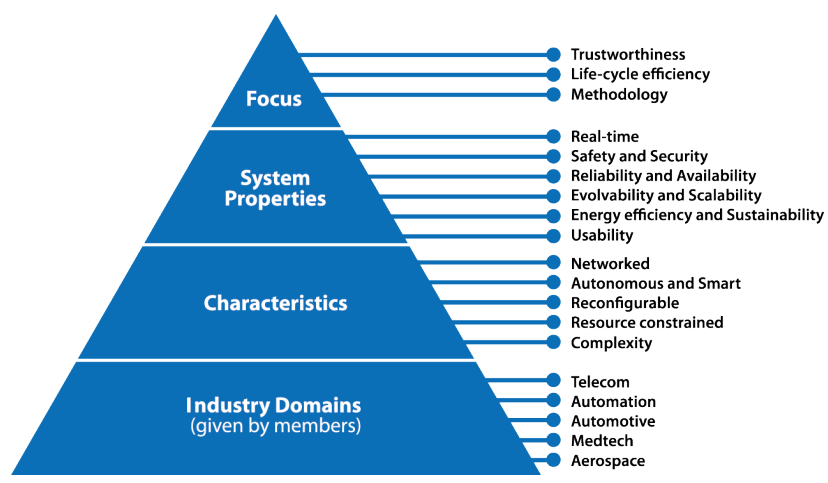

Fig. 3: Thematic focus of the ICES competence network

Operational goals and KPIs have been defined within the areas that correspond to the workgroups based on these strategic objectives, [4].

Network evolution and management. The network was officially inaugurated on September the $1^{\text {st }}, 2008$, with KTH, ABB, ÅF, Ericsson, Enea, Scania, and Stoneridge as initial members.

During its 10 years of operation, the ICES center has grown, and now has more than 30 companies as members. It has a trackrecord of actually "improving the lives" for its stakeholders. As a KTH center, ICES has a board, a director, a co-director and an administrator. Student representatives (from selected, relevant KTH programs) are part of the board to strengthen the bidirectional interactions with students (student representation is quite common at $\mathrm{KTH}$, but this case is special with its connections to multiple programs). The center is funded by the members (roughly $50 \%$ of the turnover), innovation project funding (roughly 25\%), and by KTH (roughly $25 \%$ ). The turnover is in the order of $200 \mathrm{kEUR}$, which is used to fund the ICES office (roughly one FTE) and ICES events. The actual turnover is much larger considering the in-kind efforts, which are at least in the same order of magnitude as the direct funding. Moreover, there is value created by catalyzed spin-off research projects, improvements in education, and other secondary effects caused by the networking.

ICES distributes a newsletter to some 1500 receivers and arranges a number of events each year. Most events are open. Some are free and some have a differentiated fee (with rebate for members). Generally, the fees are low and only covers the cost of the events.

The competence and work groups. A central part of the network is the industrial competence groups where industrial experts and KTH faculty meet, organize activities (such as workshops), and exchange experiences on topics of common interest (cmp. Fig. 3) and/or on education and continued education.

The current competence groups are as follows:

- $\quad$ Artificial intelligence and machine learning 
- $\quad$ Autonomous systems and platforms

- $\quad$ Embedded real-time systems

- Interoperability

- $\quad$ R\&D Managers

- Safety

- Security

- $\quad$ Testing of embedded systems

- $\quad$ Systems engineering

A new competence group usually starts by a stakeholder raising a need, followed by one or more workshops where the group is formed. This includes identifying an (initial) core team (usually composed of a mix of people from academia and industry), and deciding on an (initial) focus. The competence groups have slightly different profiles in terms of the types of activities they have chosen to organize. For example, the Safety group has emphasized the organization of a relatively new Scandinavian Conference that provides a venue for cross-domain industrial interactions and for meeting academia. The systems engineering group has instead focused on introducing systems thinking and systems engineering into a capstone course at KTH with industrial engineers as teachers. It has also organized workshops, such as on how systems engineering can deal with the complexity of future CPS.

The competence groups receive administrative support from the ICES office and a small budget. The competence groups are in turn expected to organize a few events and meetings each year and report back to the ICES board. The results from the groups are made available to the network through workshops, seminars, courses and conferences.

Another central part of the network is the workgroups. Management and development of the network takes place through the following workgroups:

- Education and student interaction (improving current engineering education, and creating contacts)

- Continued education (creating and hosting courses adapted for industry)

- Marketing and member involvement

- Competence groups (as described above)

- $\quad$ Learning network (supporting, managing and creating new competence groups)

- Interactions and catalyzing (research/innovation) projects

- Management of the center

As an example group activity, the continued education task force has been investigating prioritized thematic topics by industry, funding, and models for implementing flexible and part on-line courses. A course is now being developed in system safety and cyber-security as a collaboration between faculty from computer science and industrial engineering. This type of course is largely lacking both in engineering and continued education today.

Achievements. Among the achievements, the ICES network has catalyzed multiple research projects ${ }^{1}$, a new research center ${ }^{2}$, and annually organizes more than 20 events. As examples of such events we present an overview of events that were organized during 2017:

- Two conferences: The ICES annual conference with a focus on "DevOps for CPS", and the 5th Scandinavian Conference on System and Software Safety. Both conferences had roughly 100 participants from industry and academia each (some 60\% from industry, with a good spread across industrial domains and academic disciplines).

- $\quad 50$ work group and competence group meetings corresponding to about 3-4 meetings/year.

- Multiple efforts to promote student-industry interactions, including dedicated MSc thesis fairs, study visits, and industry involvement in teaching (e.g. guest lectures).

\footnotetext{
${ }^{1}$ Examples of catalyzed projects include the European projects iFEST (ARTEMIS-project initiated through ICES), MBAT (ARTEMIS project with ICES initiated Swedish sub-consortium) and SCOTT (ECSEL project with ICES initiated Swedish sub-consortium), each larger 3-year research projects involving multiple ICES stakeholder organizations.

2 The TECoSA research center was recently approved and is scheduled to start early 2020 . https://www.vinnova.se/en/news/2019/06/efforts-onworld-class-research-environments/ (accessed Oct. 2019).
} 
Preprint: Competence networks in the era of CPS - presented at the WESE workshop at ESWeek October 2019

- 7 workshops, for example on the topic of CPS foundations and trustworthiness, Systems engineering and CPS education needs in collaboration with INCOSE, and Innovation in CPS and IIoT (featuring funding opportunities and information from innovation support organizations).

- Several special ICES events, including the then newly started "coffee with an expert series" (featuring e.g. prominent guest professors and industrial experts), a meeting with the Swedish minister on Research, and an M.Sc. thesis fair.

- Several other events with ICES involvement, including Embedded Conference Scandinavia and the Cybersecurity and Privacy (CySeP) Summer School (co-funded by ICES).

As a further example of achievement, ICES paved the way for the international master-level program in Embedded Systems at $\mathrm{KTH}$. Given that KTH already had many programs, the industrial support for launching a new program was decisive in motivating its launch. The program was designed as a collaboration between four schools, each providing their "best courses" to the program. The interest in the program has grown from its start in 2011. 2019 the program had about 700 applicants worldwide, with 335 having the program as its first priority. To maintain top quality, the number of students has been limited to about 50-70 per year. The program scores well in course evaluations and is highly appreciated by the industrial partners in ICES.

\section{Lessons learnt}

In operating the ICES network for more than 10 years we have designed, introduced, revised and observed a number of practices that we have found especially important for the long-term evolution, growth and efficient operation of the network. These are here summarized.

Stakeholders. ICES early on spent an effort in identifying key stakeholders and their needs, recall Fig. 2. While the needs have been revised, this early effort has been instrumental in providing the relevant "services" for the stakeholders, thus creating interest in ICES. For example, it is clear that there is a large demand for CPS engineers - thus creating strong incentives for industry to engage with universities ${ }^{3}$. The paradigm shift motivates extra efforts for revising education programs. Indeed, university educators are actively engaged in discussing how to reform programs to educate the CPS engineers of tomorrow, see e.g. $[6,7,8]-$ and here it is beneficial with university and industry interactions to better understand the needs for how such new programs could be formed.

Vision and goals. In ICES, the Vision and goals document has been jointly elaborated, followed-up and improved. The operational goals, concretely drive work towards the vision and goals. Over the years, the role of ICES as a competence network has been strengthened by the insights and experiences in designing and redesigning activities to create value for the stakeholders. The name of ICES stands for Innovative Center for Embedded Systems, with "Innovative" indicating the purpose to be innovative and learning in the way the center is organized. Accordingly, we have continuously had a dialogue with stakeholders and attempted to improve a practices as well as introducing new ones.

Agreement. In the set-up of ICES, various types of agreements were considered. Eventually it was decided to not use (complex) agreements. Instead ICES relies on a code of conduct (including that the network cannot be used for marketing purposes) and the understanding that aggressive competitors are not included. However, it must be noted that many of the partners are indeed competitors (e.g. multiple consultancy companies, Scania and Volvo) but here the benefits of long-term competence development are seen as more important. Generally, information that partners bring to the network is considered to be open. This approach can be compared with research projects with rather complex contracts and with results that are not generally and widely shared. Agreements come into play for example when ICES catalyzes a research project. Such projects will then be separate from ICES with their own agreements, but can still benefit from ICES as a vehicle for bi-directional communication.

Board composition, management and decentralized organization. The ICES board has generally been rather large, involving "gold member" representatives, as well as representatives from the involved schools at KTH, with the purpose to engage the organizations and to spread information. Moreover, with the various groups, the organization is decentralized, engaging multiple persons that contribute in various ways. The management is divided further into a director and co-director, and a management team, creating robustness in the organization.

Stakeholder engagement. As part of the key stakeholders, we would like to explicitly mention the involvement of student representatives in the board and in some of the work groups. The students currently represent three relevant master programs at KTH. The students provide valuable insights into how they see the current education and improvement potential, communi-

\footnotetext{
${ }^{3}$ According to an investigation by ARTEMIS and ITEA2, the global market of Digital Technology (encompassing software, embedded software, IT services, internal IT and hardware) was estimated to USD 3,300 billion, corresponding to approximately 50 million jobs, predicted to have continued strong growth, [5].
} 
Preprint: Competence networks in the era of CPS - presented at the WESE workshop at ESWeek October 2019

cate ICES activities to students, and facilitate student interactions with industry as well as with academic faculty. As a further example, one of the competence groups is dedicated to R\&D managers so that managers with similar technology challenges but in different industry domains meet 3-4 times a year - providing interactions that are perceived as valuable by the participating managers as well as for enriching the ICES network.

From disciplinary to multidisciplinary. Based in a university, it is important to be aware of the difficulties in trying to create integration across academic disciplines, departments and schools. Universities have deep traditions and have slow time constants; for example, it will take more than one year to launch a new program and after its launch there will be several more years before the first students exit the program.

Moreover, there is a lack of incentives to integrate across disciplines. As opposed to a company that needs product integration, academic faculty are usually directly rewarded by specialization, depth and in staying within their discipline. While there might be large potential in multidisciplinary work, it takes time, is risky and sometimes even counter-productive in that funding schemes may make it difficult to include more than one discipline or more than one group from a university. Competence provisioning in the era of CPS is thus challenged by the current disciplinary university system and the growing amount of knowledge - resulting in fragmentation across disciplines, experts, groups, etc. The disciplinary setting makes it more difficult to provide new bridging competences required for future CPS and there is a corresponding lack of T-shaped educators, [9]. Similarly, academic teachers often lack first-hand experience with industrial applications, creating a gap between the taught theory vs. concerns of real CPS.

We believe that competence networks can at least partly help in addressing these problems. Establishing collaboration between academia and industry, can help to bridge the gap between theory and real-world CPS, and also incentivizes creating bridges between disciplines. ICES has contributed to improved contacts to industry for both faculty and students, and we have through the collaboration also been able to integrate industry taught modules. The purpose here has been to bring in industrial competence related to complex engineering issues that are not (readily and typically) taught at a university, complementing traditional academic courses. This has for example included the introduction of an industry taught module on systems engineering as part of a capstone course at $\mathrm{KTH},[10]$.

A competence network, with opportunities for contacts with industry and for catalyzing research projects thus helps in providing incentives towards integration. ICES has catalyzed several research projects that involve more than one research group and discipline at KTH. The era of CPS also provides new opportunities to promote multidisciplinary research since the needs are becoming much more apparent - for example, when equipping cars with various artificial intelligence (AI) techniques, safety concerns become relevant for the AI community.

Collaboration with other networks. In the area of CPS, and especially in the midst of a socio-technical paradigm shift, a huge number of efforts and centers are devoted to various aspects that relate to CPS. ICES has evolved into a networking center, establishing strategic collaboration with other initiatives as one way to deal with this plethora of initiatives. As examples of successful collaboration we would like to highlight the tradition of co-organized events with the Swedish chapter of INCOSE, and the KTH membership in ARTEMIS-IA. Currently, ICES is offering collaboration to other KTH centers that in some way relate to software-intensive CPS. The potential arises since most other centers are focused on research while ICES is a competence network. ICES also collaborates with THINGS - a KTH based CPS/IoT incubator, bringing startups and small SMEs closer to the ICES network, and thus to larger companies, KTH faculty and students.

Neutral ground and regional focus. We have noticed that having a university to host a competence network will have some form of trust enhancing effect - with the university seen as more neutral compared to many other stakeholders. ICES has further traditionally had a regional focus (the larger Stockholm area), facilitating for stakeholders to physically engage in the network.

\section{Challenges}

During the operation of the ICES network we have encountered several challenges. We here highlight what we consider as key challenges that are relevant when setting up a competence network, especially considering hosting at a University.

Competence networks and education are not prioritized. At universities, research merits are generally driving promotion (e.g. along tenure track schemes), and education has a much lower status; this is also true for continued education, [6, 11, 12].

This somewhat chocking reality has a further implication that adoption of best practices in education is progressing very slowly - much university education is still using passive learning through traditional lectures whereas there is clear scientific evidence of much improved education when active learning is adopted, [13]. 
Universities are typically given the task to communicate and share knowledge to a broader audience, referred to as public or science outreach, or the third mission of a university. Unfortunately, the third mission is vaguely defined and not incentivized. As a result, academic researchers - who are generally busy doing research within their specific discipline, will not prioritize competence network initiatives since it draws their attention from their research. When enthusiasts are recruited, on the other hand, activities may become dependent on them, making it difficult to provide continuity.

The lack of priorities for competence networks is seen for example in the lack of funding to support such activities; while research is funded, dissemination of research and exchange of best practices from industry are also not prioritized (typically with smaller short-term efforts as part of research projects). Another indication is the funding struggles we have had with ICES. Most of the centers at KTH are research centers, with larger but time-limited funds provided by e.g. public funding. For such centers, a standard model will provide funding support from the university. ICES is on the other hand a competence network that operates on lower budgets and with no clear time limit. While research centers deliver tangible research output, ICES output is often more subtle or indirect, with for example work to improve the engineering education, catalyzing new research projects and through other effects that we may not even be aware of. Capturing and estimating the corresponding indirect value is difficult. A competence network thus often represents an "odd-bird" at a university. We therefore believe that there are strong needs to balance the priorities; given the importance of competence, regions that emphasize this are likely to become winners!

More recently, the funding situation has improved due to the involvement of ICES in EU innovation projects and a Nordic academic network on the industrial internet of things, [14]. As a related opportunity and centered in ICES, a Digital Innovation Hub on Industrial Digitalization was recently formed, [31].

The paradox with strong needs but (perceived) lack of time. Industrial companies would generally agree that competence networks are increasingly important. However, at the same time, most industrial stakeholders and experts in the field perceive themselves as very busy. This is quite natural during a paradigm shift. It is however likely to create an imbalance in addressing short-term vs. long-term needs (this balance relates closely to the previous challenge, the lack of prioritization). Because people are so busy, it becomes more difficult to engage people in various competence network activities. We also see this issue with trends such as breakfast seminars, lunch meetings, webinars etc. - trying to grab the small free slots available and resorting to shorter "doses" of interactions. As a consequence, a competence network may need to spend more time on management and work to make seminars and workshops worthwhile to attend. It becomes imperative to consider the needs of stakeholders and design activities to give value to the attendees. We further believe that it is essential to develop a culture and schemes to promote the longer-term perspective, for example, by having companies invest in their employees continued education by engaging in suitable competence networks.

Communication. In these days of communication overflow, finding the right channels and being able to actually get the attention of stakeholders is becoming increasingly challenging. People are already overloaded with information, very busy (cmp. previous bullet), and various hypes makes it even more difficult to penetrate the ether. Just using email is no longer good enough. Moreover, different organizations use different IT-tools and this makes it more difficult to create for example useful forums or wiki like information exchange. ICES is currently using multiple modes of communication, including through the contacts created in the network, a newsletter, social media, and a web page where presentations, recordings, contacts etc. are made available. A special challenge is to reach out to companies which are new to CPS. Developing and implementing clear communication strategies is essential and further work is needed.

Fragmented efforts addressing competence provisioning. Different aspects of competence networks are today addressed by many initiatives and organizations. These efforts are however fragmented, there is a lack of coordination and overview of who does what, and the sharing of best practices is limited. As one remedy, ICES has initiated dialogues with other initiatives and engaged in several collaborations with other associations as described in Section 4.

\section{Discussion}

In this section we discuss our findings by relating ICES to the state of the art as described in Section 2.1, and by elaborating on the generality of our findings.

\subsection{Relating ICES to the discourse on learning networks}

We relate back to the Prioritization and "Paradox" challenges identified in Section 5 and then further discuss purposes of University and academia collaboration.

Prioritization. Related to the lack of prioritization, the incentives and evaluation of Universities today to a very little extent encompasses the third mission. Universities are today mainly evaluated as individual organizations - not relating to their interactions with other organizations, implying that University accomplishments that relate to their collaboration with compa- 
nies and other external actors are likely to be unnoticed. For individual researchers, their research production represents the main incentive. For the University as a whole, the main incentives in Sweden today include funding related to the educational output ("finished students") and the "ranking" which is geared towards research performance.

With respect to companies, they often invest too little in competence development and instead focus on having a higher employee turnover to "shift competences". In reality this is most likely quite costly, but it is easier to argue inside a firm that new employees with desired competence should be hired while others lacking the right knowledge and skills should be laid off than to argue that employees need a budget for lifelong learning. One reason for this may be that central HR takes much of the costs for hiring and firing, whereas continuous competence development is typically carried out by the business units, resulting in unbalanced incentives. Companies need to consider the real costs of updating competences as compared to hiring new people, and maybe also policy makers need to create incentives for lifelong learning inside companies, not only support activities for the ones who have been found to have outdated knowledge and consequently have lost their jobs.

Paradox and communication. During the years with ICES, we see a lot of potential for even more engagement by the involved companies. Apart from "the lack of time" that we perceived, the state of the art provides an additional explanation the cost of interacting with others with a different background. Without the strong motivation that comes from working towards direct value capture or knowledge creation the participants thus struggle to find the time to engage continuously. The paradox also relates closely to the positioning and culture within the company (as just discussed under priorities) - and this requires some level of management support and a way of operating the network, and of course, interest by people.

The barrier for engaging may also relate to the fact that competence networks constitute unusual entities (i.e "odd birds" as noted in Section 5) in the world of academia, Comparing with traditional research centers, ICES offers a slightly more complicated mode of engagement. In the setting of a research center, a company pays money to the center, and is typically in return invited to a few workshops per year and a committee for deciding on projects. ICES on the other hand offers rich opportunities for interactions in terms of the work groups and competence groups, the board, workshops, conferences, and other activities. To access the network thus requires an initial effort in understanding what the network and groups are about, and then further time (and prioritization) in actually engaging.

The paradox further relates to the challenge of communication. To reduce the barriers, there is a need for efficient communication. We believe that IT-support in various forms (from webexes, recorded talks, forums etc.) have a role to play but deploying and getting the network to adopt the right tools also introduces another potential barrier so this requires careful considerations. W.r.t. communication we have noticed that our reach within some of the larger company members is somewhat limited. To spread information in such large companies, we believe it is beneficial to tap into their communication structures. Such efforts have been attempted and will be continued in ICES.

A competence center can in many ways be seen as a "community of practice" as it is based on some important pillars: a shared view of its purpose (learning), mutual trust (making it possible to reveal both strengths and weaknesses), efficient communication through specialized language (jargon), a strong focus on knowledge (meritocracy) rather than power stemming from hierarchical positions, and a network-based organization. In order to function as a community, it cannot be expanded to a very large group of people as this will undermine all the above pillars, and consequently this type of community has to be established and managed locally in a bottom-up way (not excluding support from university and funding bodies, but these should ideally not come with too much demands and limitations).

Purposes of University and academia collaboration. The role of academia in supporting industry and society encompasses education, research and outreach.

Ensuring a continued flow of possible recruits with the right knowledge is in itself a strong motivator for public-private cooperation. Our experiences from ICES suggest that this type of cooperation can also affect the involved stakeholders in other ways than e.g. cooperation focused on innovation: understanding each other, achieving a common vision and finding a neutral common ground was strongly emphasized among the stakeholders.

Interactions taking place in competence networks provides information about graduates' needed competences and skills as this is made explicit by industry representatives, making it possible to adjust curricula accordingly in order to increase employability and reduce the time needed for graduates to find suitable employment. The high cost of misalignment between supply and demand for university-developed knowledge and skills could thus be reduced by access to more relevant and updated information about needs and development trends. In our experience, this type of collaboration has a strong potential since in the era of complex CPS - insights into technology, engineering complex systems and science will all be required. The collaboration has to respect the integrity of the involved organizations, and balance short vs. long term as well as between science, engineering and technology. 
Preprint: Competence networks in the era of CPS - presented at the WESE workshop at ESWeek October 2019

\subsection{Generality of findings}

If one would like to replicate the ICES competence network - how would that work in other regions and countries, i.e. what might be specific for the regional context of ICES?

The operation of the ICES competence network has evolved in Sweden in the larger Stockholm area. We believe that many of the experiences are valid also in other settings, although differences in regional needs, constraints and culture needs to be considered in attempting to draw upon the findings from ICES.

The Swedish setting is characterized by free university education, a strong multi-domain industry, and a tradition of governmental sponsoring of research and education (with little corresponding direct industrial sponsoring of such activities). In addition, Sweden is known to be home to a collaborative culture. Moreover, the use of the university as neutral ground, the policy to avoid fierce competitors, and the regional scale have most likely been important in creating an environment of trust, where no agreements are needed and where people are still participating and sharing information.

Thus, while we do believe that many of the ingredients and effects as discussed in Sections 4 and 6.1 should be relevant in other regions with other characteristics, the element of building trust will be important for setting up a competence network also in other contexts.

We note that the trends and challenges with CPS are not specific to any region. The industrial presence and the types of domains related to a region could however vary. A broader spectrum of CPS application domains - such as we are fortunate to experience with ICES, certainly implies that it should be easier to set up a competence network. Engaging in a network to share experiences, may be easier across domains since there is less competition. However, even with a smaller number of domains, the needs for competence networks are still strong.

Many other areas are also characterized by complex systems and paradigm shifts, involving a range of stakeholders. We believe that the experiences from the ICES network could be relevant in other domains, beyond CPS. For example, considering the transitioning to sustainable and circular systems, it would be highly beneficial to establish collaboration - e.g. in the form of a competence network - involving for example stakeholders representing economy, management, material scientists, experts in sustainability (e.g. life-cycle analysis), industrial manufacturing, systems engineering, and digital technology experts.

\section{Conclusions and ways forward}

The amount of knowledge required in developing and managing the CPS of tomorrow is unprecedented and requires grasping a broader area of concerns and a corresponding availability of experts in these areas. This leads to strong needs for collaboration.

Competence networks address the needs for CPS involved organizations to continuously learn within and across organizations, to get access to experts and recruit competent people, in sharing experiences and best practices, and working to re-shape the engineering education to better meet the needs for engineering future CPS, including developing life-long learning through continued education for the existing work-force.

We have described the ICES competence network involving academia and industry, lessons learnt and challenges faced during the operation of the network. There are several important ways forward:

- Competence networks ought to be recognized as important mechanisms for spurring regional innovation. In order to accomplish this, incentives, funding, KPIs and evaluation schemes need to be made available. The EC initiative on digital innovation hubs could here correspond to one useful mechanism forward.

- Companies needs to recognize the importance of internal competence development, and here collaboration with universities in the form of competence networks represents one promising way forward.

- As well recognized, but still pertinent to this paper, there is a need to provide incentives for life-long learning and to raise the status of education

- Gathering, investigating and disseminating best practices on competence networks. We believe that further work is needed in this direction, including addressing the identified challenges.

In conclusion, our experience is that the ICES type of competence network fills an important role as information carrier and collaboration mechanism in our regional setting with cross- industry domain and academia collaboration. The network is perceived as important by the involved stakeholders, and would be relevant to introduce also in other areas involving complex systems. 
Preprint: Competence networks in the era of CPS - presented at the WESE workshop at ESWeek October 2019

\section{Acknowledgments}

This work has been supported by FED4SAE (H2020 Innovation action), HI2OT (supported by NordForsk's Nordic University Hubs programme, grant agreement no. 86220) and ICES at KTH. We greatly acknowledge the contributions of the many persons and organizations that have engaged in ICES or otherwise supported ICES since its start in 2008!

\section{References}

1. Haydn Thompson et al. Platforms4CPSKey Outcomes and Recommendations. Report from the Platforms4CPS project (H2020 project Grant Agreement No 731599) - 1st edition, 2018 | Steinbeis-Edition, StuttgartISBN 978-3-95663-184-9.

2. Martin Törngren and Paul T. Grogan. How to Deal with the Complexity of Future Cyber-Physical Systems?, Journal of Designs, Vol. 2 (4), 2018, DOI: 10.3390/designs2040040.'

3. ICES web page: www.ices.kth.se (referenced 2019-08-12)

4. ICES - VISION and GOALS; Martin Törngren et al., Feb. 2018 - ICES working document, available from [3].

5. ITEA ARTEMIS-IA High-Level Vision 2030: Opportunities for Europe, Autumn $2013 . \quad$ URL: https://itea3.org/publication/download/itea-artemis-ia-high-level-vision-2030-v2013.pdf (accessed 2019-08-12)

6. Martin Törngren, Saddek Bensalem, John McDermid, Roberto Passerone, Alberto Sangiovanni Vincentelli and Bernhard Schätz. Education and training challenges in the era of Cyber-Physical Systems: beyond traditional engineering. Workshop on Embedded and Cyber-Physical Systems Education (WESE) at ESWEEK 2015, Amsterdam. http://dl.acm.org/citation.cfm?id=2832928

7. First Workshop on CPS Education, April 8th, 2013, Philadelphia, PA (part of CPSWeek 2013) - accessible at http://cpsvo.org/group/edu/workshop (accessed July 2015).

8. Workshop on Embedded and Cyber-Physical Systems Education (WESE), 2015, Organized as a part of Embedded Systems Week, http://www.emsig.net/conf/2015/wese/

9. Martin Törngren and Erik Herzog. Towards integration of CPS and Systems engineering in education. Proc. 2016 Workshop on Embedded and Cyber-Physical Systems Education, 12th Embedded system week, Pittsburgh, Oct. 2016.

10. Erik Herzog, ÅN Larsson, J El-Khoury, M Törngren. Experience from Introducing Systems Engineering in an Aca-demic Environment Using an Industry Training Course. INCOSE International Symposium 28 (1), 245-259, 2018.

11. Debate article in Dagens Nyheter (Swedish newspaper ) by Swedish University Chancellor Harriet Wallberg, 2014-08-04 on "Unacceptable de-prioritization of the higher education system" (article in Swedish): http://www.dn.se/debatt/oacceptabel-bantning-avundervisning-pa-universitet/ (accessed August 2019)

12. Chalmers, Denise. Progress and challenges to the recognition and reward of the scholarship of teaching in higher education. Higher Education Research \& Development

13. Michael Prince. Does Active Learning Work? A Review of the Research. Journal of Engineering Education, July 2004.

14. http://www.nordic-iot.org/ (accessed August 2019)

15. Grant, R. M. (1996). Toward a knowledge-based theory of the firm. Strategic management journal, 17(S2), 109-122.

16. Ozman, M. (2009). "Inter-firm networks and innovation: a survey of literature." Economic of Innovation and New Technology 18(1): 39-67.

17. Pyka, A. (2002). "Innovation networks in economics: from the incentive-based to the knowledge-based approaches." European Journal of Innovation Management 5(3): 152-163.

18. Ankrah, S. and A.-T. Omar (2015). "Universities-industry collaboration: A systematic review." Scandinavian Journal of Management 31(3): 387-408

19. Mansfield, E. (1995). "Academic research underlying industrial innovations: sources, characteristics, and financing." The review of Economics and Statistics: 55-65.

20. Lin, J.-Y. (2017). "Balancing industry collaboration and academic innovation: The contingent role of collaboration-specific attributes." Technological Forecasting and Social Change 123: 216-228.

21. Cohen, W. M. and D. A. Levinthal (1990). "Absorptive Capacity: A New Perspective on Learning and Innovation." Administrative science quarterly $35(1): 128-152$.

22. Hess, A. M. and F. T. Rothaermel (2011). "When are assets complementary? Star scientists, strategic alliances, and innovation in the pharmaceutical industry." Strategic management journal 32(8): 895-909.

23. Soh, P.-H. and A. M. Subramanian (2014). "When do firms benefit from university-industry R\&D collaborations? The implications of firm R\&D focus on scientific research and technological recombination." Journal of Business Venturing 29(6): 807-821.

24. Jamieson, L. H. and J. R. Lohmann (2012). Innovation with impact: Creating a culture for scholarly and systematic innovation in engineering education. Washington, DC, American Society for Engineering Education. 
Preprint: Competence networks in the era of CPS - presented at the WESE workshop at ESWeek October 2019

25. Kasworm, C. and L. Hemmingsen (2007). "Preparing professionals for lifelong learning: Comparative examination of master's education programs." Higher education 54(3): 449-468.

26. Seely, B. (2005). Patterns in the History of Engineering Education Reform: A Brief Essay. Educating the Engineer of 2020: Adapting Engineering Education to the New Century. Washington, DC, The National Academies Press: 114-130.

27. Downey, G. L. and J. C. Lucena (2004). "Knowledge and professional identity in engineering: code-switching and the metrics of progress." History and Technology 20(4): 393-420.

28. Corbett, A. (2012). Principles, Problems, Politics ... What Does the Historical Record of EU Cooperation in Higher Education Tell the EHEA Generation? European Higher Education at the Crossroads. Dordrecht, Springer: 39-58.

29. The Magna Charta Observatory (1988). Magna Charta Universitatum. Bologna, Bologna University.

30. Herbert A. Simon (1987). The steam engine and the computer: what makes technology revolutionary. Computers and People 36, 1112 (November 1987), 7-11.

31. The KTH Innovation Hub of Digital Industrialization - https://s3platform.jrc.ec.europa.eu/digital-innovation-hubs-tool//dih/5792/view (accessed 2019-10-12).

32. David Patterson (2013). How to Build a Bad Research Center. Technical report UCB/EECS-2013-123, EECS Department, Univ. of California, Berkeley, June, 2013. http://www2.eecs.berkeley.edu/Pubs/TechRpts/2013/EECS-2013-123.html (accessed 2019-10-12).

33. Brown JS, Duguid P. 1991. Organizational learning and communities-of-practice: toward a unified view of working, learning, and innovation. Organization Science 2(1): 40-57.

34. Wenger E. 2001. Communities of practice and social learning systems. Organization 7(2): 225-246. 